\title{
The Environment of the Young Earth in the Perspective of An Young Sun
}

\author{
Vladimir S. Airapetian \\ NASA GSFC, Code 671, Greenbelt, MD, USA \\ email: vladimir.airapetian@nasa.gov
}

\begin{abstract}
Our Sun, a magnetically mild star, exhibits space weather in the form of magnetically driven solar explosive events (SEE) including solar flares, coronal mass ejections and energetic particle events. We use Kepler data and reconstruction of X-ray and UV emission from young solar-like stars to recover the frequency and energy fluxes from extreme events from active stars including the young Sun. Extreme SEEs from a magnetically active young Sun could significantly perturb the young Earth's magnetosphere, cause strong geomagnetic storms, initiate escape and introduce chemical changes in its lower atmosphere. I present our recent simulations results based on multi-dimensional multi-fluid hydrodynamic and magnetohydrodynamic models of interactions of extreme CME and SEP events with magnetospheres and lower atmospheres of early Earth and exoplanets around active stars. We also discuss the implications of the impact of these effects on evolving habitability conditions of the early Earth and prebiotic chemistry introduced by space weather events at the early phase of evolution of our Sun.
\end{abstract}

Keywords. The Sun, Earth, space weather, CME, SEP, atmosphere, chemistry, exoplanets, active stars

\section{Introduction}

The early Earth in the late Hadean period was a highly energetic and dynamic planet. Despite of hellish conditions introduced by intensive volcanic and tectonic activity, frequent impacts by Late Heavy Bombardment events and high fluxes of X-ray and UV radiation from the early Sun as well as frequent eruptive ejections from young Sun, liquid water was an essential ingredient of our planet in its earliest history (Wilde et al. 2001; Gomes et al. 2005; Abramov \& Mojzsis 2009; Airapetian et al. 2016). Recent biogenic carbon data suggest that our young planet in the first 0.7 Gyr managed to support the initiation and development of life (Bell et al. 2015). The conditions controlling life include of the appropriate surface temperature and pressure to support liquid water and biochemical processes on the early Earth along with X-ray \& UV fluxes and solar wind energy fluxes from the young Sun. Geophysical factors include internal dynamics between the inner and outer Earth core driving generation of geomagnetic field and the magnetosphere and plate tectonics and volcanic processes that contributed to mantle degassing and atmosphere refueling. The geodynamo has been operating over last 4 billion years and provided the magnetic shield, which was strong enough to withstand the pressures from the young Sun wind (Tarduno et al. 2015).

What combination of geophysical and astrophysical conditions driven by the internal dynamics of the Earth and activity of the young Sun create habitability factors favorable for initiation of life? This is one of the most fundamental questions of the modern science because the answer to this question will provide a unique opportunity to understand how life might form on other planets and how to search for their observational signatures. 
In this review, I describe our recent progress in understanding space weather from the current and the young Sun at the time when life started on Earth and discuss physical processes that drive their interactions with out planet. Here, I emphasize the negative and positive factors affecting habitability conditions on the young Earth as an important test case for understanding the habitability conditions on terrestrial type exoplanets around main-sequence stars of $\mathrm{G}, \mathrm{K}$ and $\mathrm{M}$ spectral classes recently discovered by Kepler Space Telescope, HST and ground-based telescopes. Finally, I discuss the role of space weather processes in initiation of prebiotic chemistry that set favorable conditions for the origin of life on the young Earth.

\section{The Activity of the Young Sun}

Recent X-ray and UV missions including CHANDRA, XMM-NEWTON, the Hubble Space Telescope and recent Kepler Space telescope opened new windows in studying the lives of stars resembling our Sun at various phases of evolution. These observations provided a unique opportunity to trace the properties of activity of the young Sun by observing other young solar-type stars. This may provide crucial information in our understanding of habitability of the early Earth, Mars and Venus and factors controlling the origin of life on our planet.

In order to reconstruct the properties of explosive events from the young Sun, we need to examine observations of young solar-like stars resembling our Sun in its infancy. Observations of young (a few hundred Myr old) solar-like stars show that our Sun had about $30 \%$ less luminous (at the time when life arose on our planet) due to less dense core driven by thermonuclear fusion of hydrogen into helium (Gough 1981).

As Sun evolves, its luminosity increases roughly $10 \%$ per every billion years. Despite its lower bolometric luminosity, the young Sun represented a very magnetically active and rapidly rotating star. Rapid rotation in combination with deep convection zones of these stars produce strong surface magnetic field that emerge to the surface and form compact, dense and hot corona (10 MK) of the young Sun. Recent direct measurements of surface magnetic fields from young suns (Bcool project) shows that the surface magnetic flux of an $0.5 \mathrm{Myr}$ old star is by a factor of 30 greater than that measured from the current Sun as a star (Vidotto et al. 2015). Such strong magnetic fields serve as the major energy source to produce frequent and energetic flares in their coronae contributing to plasma heating and production of large X-ray luminosities that by 3-4 orders of magnitude greater than that observed today (Pevtsov et al. 2003; Gudel et al. 1997; Tu et al. 2015).

The photospheric convection motions excite Alfvèn waves that propagate upward and contribute to the initiation of the fast solar and dense solar wind. The winds from young active sun can play a crucial role in removal of angular momentum from them and resulting in spin-down in times as stars age (Sterenborg et al. 2011; Garraffo et al. 2016). We have recently used a three-fluid three-dimensional magnetohydrodynamic Alfven wave driven solar wind model, ALF3D, to study the evolution of the young solar wind. Our model treats the wind thermal electrons, protons and pickup protons as separate fluids and incorporates turbulence transport, eddy viscosity, turbulent resistivity, and turbulent heating to properly describe proton and electron temperatures of the solar wind. We used three input model parameters, the plasma density, Alfven wave amplitude and the strength of the magnetic dipole field at the wind base for each of three solar wind evolution models. We concluded that the terminal velocity of the young solar wind was twice faster, 100 times denser and 5 times hotter at 1 AU in its early history (at 0.7 Gyr) (Airapetian \& Usmanov 2016). 


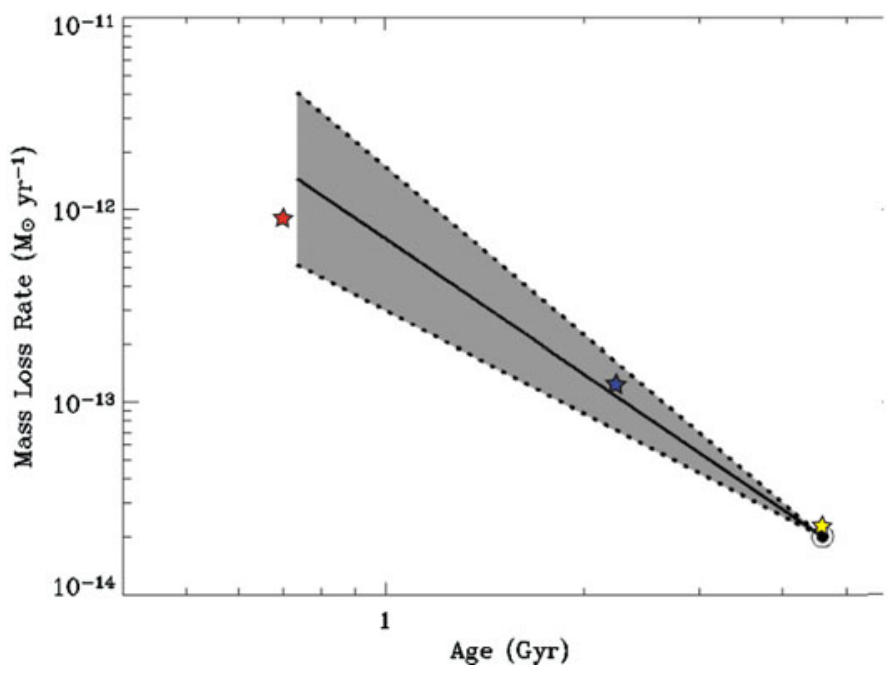

Figure 1. The total mass-loss rates from the solar wind at 0.7 Gyr (red star), 2.2 Gyr (blue star) and 4.6 Gyr (yellow star) superimposed on the empirically derived values of mass loss rates (grey area) from a sample of solar-type stars of various ages.

Figure 1 shows the total mass-loss rates from Sun at at the three evolutionary phases of the Sun, 0.7 Gyr, 2 and 4.65 Gyr (the current epoch) superimposed on the range of empirically derived mass-loss rates for solar-like stars at various phases of evolution (Wood et al. 2005). The evolution of the solar wind was driven mostly by the coronal magnetic field, the plasma density at the wind base and the amplitude of Alfven waves. Our models suggest that the dynamic pressure from the young solar wind at 0.7 Gyr is expected to be up to 170 times greater than the wind pressure from the current Sun.

Frequent flares on the young Sun were the source of fast and dense CMEs forming as a result of the global restructuring of the solar coronal magnetic field. The frequency of CMEs from young Sun and other active stars can be estimated from their association with solar/stellar flares. Recent SOHO/LASCO and STEREO observations of energetic and fast (> $1000 \mathrm{~km} / \mathrm{s}$ ) CMEs show strong association with powerful solar flares (Yashiro \& Gopalswamy 2009; Aarnio et al. 2011; Tsurutani \& Lakhina 2014). This empirical correlation established for the events from the current Sun provides an estimate for CME occurrence frequencies. Kepler data suggest that stellar superflare events with energy of $3 \times 10^{33}$ ergs (referred to as superflares) occur on young and active K-G type main-sequence stars at the rate of $\sim 250$ events/day (Maehara et al. 2012; Shibayama et al. 2013; Airapetian et al. 2016). This suggests that CME events associated with such superflares (referred to as super-CME events) should have the kinetic energy by a factor of 10 greater than the energy of associated flare events. The frequency of such events directed toward the young Earth is estimated to be at least a few events per day.

Another evidence for the presence of high frequency energetic flares from the young Sun comes from our direct comparison of the reconstructed X-ray to UV flux (XUV) for the young Sun using k1 Cet as a proxy for it (Airapetian et al. 2017). In Figure 2 we present the reconstructed spectral energy distribution (SED) of the current Sun at the average

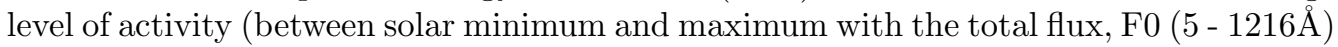
$=5.6 \mathrm{erg} / \mathrm{cm}^{2} / \mathrm{s}$; yellow dotted line), the X5.5 solar flare occurred on March 7, 2012 (blue line), the young Sun at 0.7 Gyr (yellow solid line) and an inactive M1.5 red dwarf, GJ 832 (red line). The spectra for the current Sun and the solar X5.5 flare in the XUV band (0.5$10 \AA$ ) are constructed from the Solar Dynamic Observatory (SDO)/EVE instrument data. 


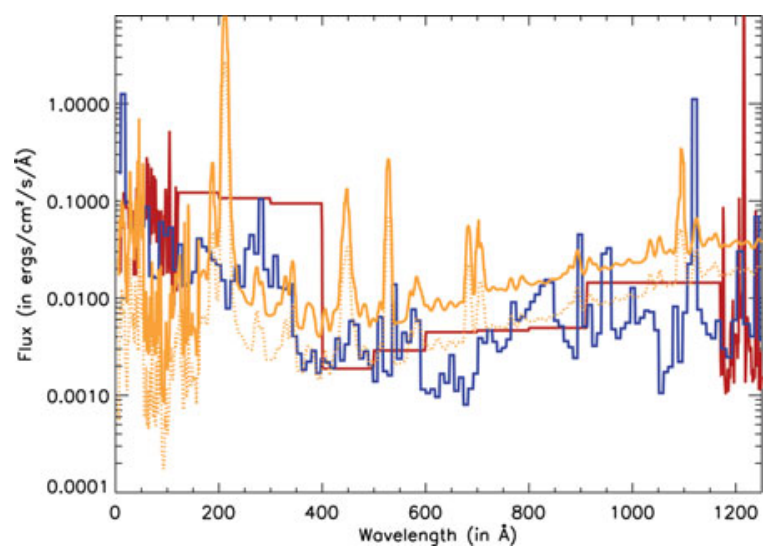

Figure 2. The spectral energy distribution (SED): reconstructed for the solar X5.4 flare (blue curve), the young Sun's SED (orange curve) and the quite Sun at the average (intermediate between solar minimum and maxium) magnetic activity (dotted orange curve) scaled to $1 \mathrm{AU}$ and GJ 832 SED (red curve) scaled to $0.16 \mathrm{AU}$

The Vacuum Ultraviolet (VUV) contribution of the total radiative output is obtained by implementing the Flare Irradiance Spectral Model (FISM, Chamberlin et al. 2008), which represents an empirical model developed for space weather applications that estimates the solar irradiance at wavelengths from 1 to $1900 \AA$ at $10 \AA$ resolution with a time cadence of $60 \mathrm{~s}$. We also reconstructed the XEUV spectrum of a moderately-old and inactive M1.5 dwarf, GJ 832, that hosts a super-Earth planet at 0.16 AU using the Measurements of the Ultraviolet Spectral Characteristics of Low-mass Exoplanetary Systems (MUSCLES) Treasury Survey data (Loyd et al. 2016). Finally, to approximate the spectrum of the young Sun at $0.7 \mathrm{Gyr}$, we used the data obtained from the parameterization of the two young solar analogs of the Sun at around 0.7 Gyr, k1 Cet and EK Dra (Claire et al. 2012). The total XEUV flux from the young Sun and the red dwarf are $8.3 \mathrm{~F}_{0}$ (at $1 \mathrm{AU}$ ) and $7.7 \mathrm{~F}_{0}$ (at $0.16 \mathrm{AU}$ ) respectively. The XEUV flux from the young Sun, and GJ 832 are comparable in magnitude and shape at wavelengths shorter (and including) Ly-alpha emission line. This suggests contribution of X-type flare activity flux is dominant in the "quiescent" fluxes from the young Sun and inactive M dwarfs.

\section{Astrophysics of Habitability of the Early Earth}

Recent paleomagnetic observations suggest that the geodynamo has been active over at least the past $3.5 \mathrm{Ga}$ (Biggin et al. 2011) and possibly even as early as $4.2 \mathrm{Ga}$ (Tarduno et al. 2015). However, the early Earth's magnetic field is expected to be weaker by an up to $50 \%$ during the Archean, while other researchers suggested a magnetic field of a quarter of the present-day intensity (Miki et al. 2009). As discussed in the previous sectons, fast and dense winds and energetic CMEs associated with superflares from the young magnetically active Sun should have exerted larger dynamic pressures on weak Earth's magnetosphere and generate energy flux at the magnetopause that may cause the atmospheric erosion. The XUV fluxes from the young Sun in the first 0.5 Gyr should have been at least 10 times of the present day solar flux (see Figure 3). Such large dynamic pressures and XUV fluxes could have ignited significant atmospheric escape from the early Earth (Airapetian et al. 2017). Indeed, recent fossilized raindrop imprint data suggest that atmospheric pressure of the Earth 2.7 Gyr ago was at least the half of 
the current pressure (Som et al. 2016). Such low atmospheric pressure creates problems in explaining the existence of oceans on Earth as early as $4.4 \mathrm{Gyr}$ ago as supported by recent zircon data (Wilde et al. 2001). This problem is further challenged by the faint (30\% less bright) young Sun at that time that makes is difficult to find an efficient warming mechanism to support liquid surface water on the early Earth. In this Section, we discuss physical processes responsible for atmospheric escape from the early Earth and ways to resolve the FYS paradox.

\subsection{Effects of super CMEs on the early Earth's magnetosphere}

As Interplanetary CMEs (ICMEs) propagate toward the Earth, they interact with Earth magnetosphere compressing its dayside and night sides. If the interplanetary magnetic field (IMF) is directed southward (or oppositely directed to the Earth's dipole field), then CMEs trigger geomagnetic storms due to the combined effects of magnetic reconnection on the dayside (as recently directly observed by MMS mission observations, Birch et al. 2016) and dynamic pressure effects (Birch et al. 2016). Also, CMEs perturb the nightside geomagnetic field producing magnetic reconnection in the Earth's magnetotail (Zhao et al. 2016).

One of the strongest CME events characteristic of the young Sun's conditions had occurred on July 23-24 2012. This event was observed as a series of two successive CME events. The first CME on July 23 had the peak speed over $2500 \mathrm{~km} / \mathrm{s}$ with the peak southward magnetic field $\mathrm{Bz}=-201 \mathrm{nT}$ (Riley et al. 2016). This catastrophic event was comparable in its energy to the kinetic energy of the Carrington event of Sep 1-2, 1859. This rare type energetic event missed the Earth. The modeling by Ngwira et al. 2013 using SWMF at CCMC/GSFC suggests that if these events would have hit the Earth magnetosphere, the stand-off distance would have been as low as $2 \mathrm{RE}$. The height integrated Joule heating rate deposited in the Earth thermosphere widened polar regions would have been as high as $2.5 \mathrm{~W} / \mathrm{m}^{2}$ or by a factor of 50 greater than in St. Patrick event. This would suggest that the temperature increase and the thermospheric expansion to at least $100,000 \mathrm{~K}$ at $150 \mathrm{~km}$. Our estimates of the frequency of such events suggest that they would have hit the magnetosphere of the young Earth 4 billion years ago at a rate of few events per day (Airapetian et al. 2015).

Here we present our results of the SWMF/CCMC based simulations of an extreme CME event referred to as a super-Carrington event interacting with magnetosphere of the young Earth. To characterize this event, we utilized the Space Weather Modeling Framework (SWMF) available at Community Coordinated Modeling Center (CCMC) at NASA Goddard Space Flight Center (see at http://ccmc.gsfc.nasa.gov). A single-fluid, time dependent fully non-linear 3D magnetohydrodynamic (MHD) code BATS-R-US (Block-Adaptive-Tree Solar-wind Roe-type Upwind Scheme) is a part of SWMF and was developed at the University of Michigan Center of Space Environment Modeling (CSEM). The spine of the SWMF is the BATS-R-US code (Powell et al. 1999). The global magnetosphere model is coupled to the inner magnetosphere through the Rice Convection Model (De Zeeuw et al. 2004). Field-aligned currents, Jpar, calculated at the lower boundary are mapped to the ionospheric height of $110 \mathrm{~km}$ under the assumption of a dipole magnetic field. From the electric currents, J, mapped at the lower ionospheric boundary the conductance obtained in the inner magnetosphere model, we calculate the Joule heating $(\mathrm{JH})$ at $110 \mathrm{~km}$.

For a super-Carrington event, we implemented the model of the young solar wind discussed in Airapetian and Usmanov (2016). The simulations were carried out using a block adaptive high resolution grid with the minimum cell size of $1 / 16 R_{E}$. The inner boundary is set at $1.25 \mathrm{R}_{E}$. The young solar wind conditions are set at the upstream 


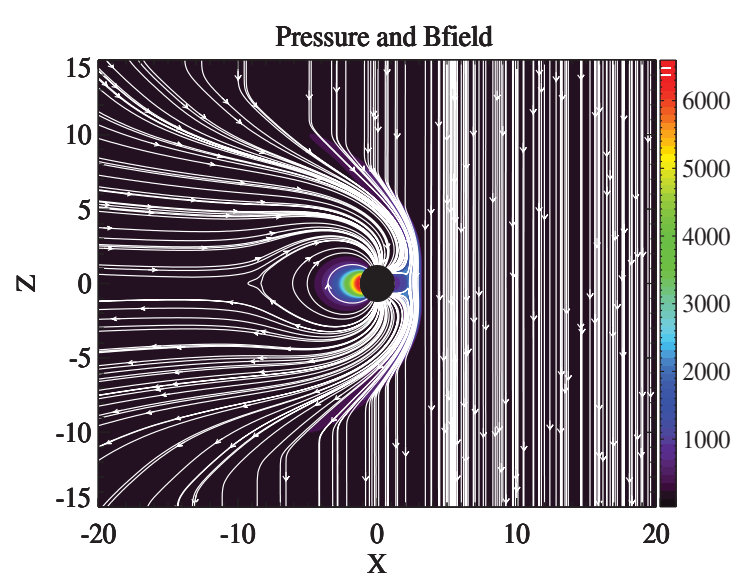

Figure 3. Magnetospheric storm at $\mathrm{t}=10 \mathrm{~h}$. Plasma pressure and the magnetic field lines. $\mathrm{X}$ and $\mathrm{Y}$ axis are given in the units of Earth radius.

boundary and some period of local time stepping is used to get an initial steady state solution.

We assume the solar wind input parameters including the three components of interplanetary magnetic field, $\mathrm{Bx}, \mathrm{By}$ and $\mathrm{Bz}$, the plasma density and the wind velocity, $\mathrm{V}_{x}$ using the physical conditions associated with a Carrington-type event as discussed by Tsurutani et al. (2003) and Ngwira et al. (2014) and Airapetian et al. (2016). Figure 3 presents a 2D map of the steady-state plasma density superimposed by magnetic field lines for the magnetospheric configuration in the $\mathrm{Y}=0$ plane corresponding to the initial 30 minutes of the simulations, when the Earth's magnetosphere was driven only by dynamic pressure from the solar wind. At $\mathrm{t}=30 \mathrm{~min}$, we introduce a super-Carrington CME event characterized by the time profile of $\mathrm{Vx}$ as the CME approaches the Earth at the maximum velocity of $1800 \mathrm{~km} / \mathrm{s}$. The CME magnetic field is directed southward or is sheared by 180 degrees with respect to the dipole field with the $\mathrm{B}_{z}=-212 \mathrm{nT}$.

As the CME propagates inward, its large dynamic pressure compresses and convects the magnetospheric field inducing the convective electric field. It also compresses the nightside magnetosphere and ignites magnetic reconnection at the nigh-side of the Earth's magnetosphere causing the magnetospheric storm as particles penetrate the polar regions of Earth. Another effect appears to be crucial in our simulations. The strong sheared magnetic field on the dayside (sub-solar point) of Earth is also subject to reconnection, which dissipates the outer regions of the Earth's dipole field up until $1.5 \mathrm{R}_{E}$ above the surface. The boundary of the open-closed field shifts to 36 degrees in latitude.

The CME drives large field aligned electric currents that provide a Joule ionospheric heating at $110 \mathrm{~km}$ reaching $4 \mathrm{~W} / \mathrm{m} 2$. The more extreme geomagnetic events introduced by stellar winds and CMEs around magnetically actiove $\mathrm{M}$ dwarfs can introduce much stronger currents in Earth-like exoplanetary ionospheres with the Joule heating rates as high as $10 \mathrm{~W} / \mathrm{m}^{2}$ (Cohen et al. 2014).

The thermospheric temperature of Earth in the quiet state at $150 \mathrm{~km}$ is $900 \mathrm{~K}$. From the first law of thermodynamics we an obtain the high estimate on the temperature change

$$
J H=\frac{7}{2} m_{H} k_{B} \frac{d T}{d t}
$$

where $\mathrm{JH}$ is the volumetric heating rate. Assuming the height of the thermosphere of $150 \mathrm{~km}$, we can derive the temperature rate change at 13,600 K/day at the heating 
rate of $0.04 \mathrm{~W} / \mathrm{m}^{2}$. However, the part of the energy we be spent to drive the thermospheric expansion that will increase the thermospheric density. Such energy deposition will induce the $\mathrm{NO}$ (at $5.3 \mu \mathrm{m}$ ) and $\mathrm{CO} 2$ (at $15 \mu \mathrm{m}$ ) mediated radiative cooling of the thermosphere, which is scaled linearly with the temperature increase (Weimer et al. 2015). Recent observations suggest that strongest storms ignite thermospheric overcooling due to such effects (Knipp et al. 2017). One can see that if the dissipation rate becomes 100 times greater, this will heat the thermosphere to huge temperatures and ignite adiabatic expansion of ionospheric plasma driving atmospheric evaporation and radiative cooling by $\mathrm{NO}$ and $\mathrm{CO} 2$. These processes will be simulated in the near future using our ionosphere-thermosphere code (Smithro and Sojka 2005). Ionospheric heating develops large gradients of plasma pressure in addition to forces which drive mass outflow at velocities greater than $20 \mathrm{~km} / \mathrm{s}$. This is greater than the Earth's escape velocity, and thus, this bulk flow contributes to the mass loss during the storm. Ionospheric cross cap potential drives large energy flux of non-thermal precipitating electrons $24 \mathrm{erg} / \mathrm{cm}^{2} / \mathrm{s}$ with the mean energy of $5 \mathrm{keV}$. These processes are crucial factors that could contribute to habitability conditions on early Earth and Mars and exoplanets around active stars.

\subsection{XUV Driven Atmospheric Escape From the Young Earth}

We have recently modeled the effects of X-ray and UV (XUV) radiation from the young Sun on atmospheric escape from the 0.7 Gyr young Earth, when XUV fluxes were by a factor of 10 greater than that at the current epoch (Airapetian et al. 2017a). XUV radiation induces non-thermal heating via photo-absorption and photoionization raising the temperature of the exosphere, and therefore, its pressure scale height. At high XUV fluxes, this process initiates hydrodynamic atmospheric escape of neutral atmospheric species, with the loss rate dependent on the molecular mass of atmospheric species. Hydrogen, as the lightest component, escapes more readily than any other species by this mechanism (Lammer et al. 2008; Tian et al. 2008). For the environments of active solartype stars, much of the hydrogen likely escapes from a planet's atmosphere during the system's early evolution, leaving behind an atmosphere enriched in heavier elements such as $\mathrm{N}$ and $\mathrm{O}$. Thus, processes of atmospheric ionization and loss via non-thermal mechanisms are crucial for modeling the evolution of oxygen and nitrogen-rich atmospheres as well the efficiency of atmospheric loss of water as a critical factor of habitability of the young Earth. In the region above an Earth-size planet's exobase, the layer where collisions are negligible, the incident XUV flux ionizes atmospheric atoms and molecules and produces photoelectrons. The upward propagating photoelectrons outrun ions in the absence of a radially directed polarization electric field and forms the charge separation between electrons and atmospheric ions. Thus, a radially directed polarization electric field is established that enforces the quasi-neutrality and zero radial current. For ionospheric ions with energies over $10 \mathrm{eV}$, the polarization electric field cancels a substantial part of the Earth's gravitational potential barrier, greatly enhancing the flux of escaping ions and forming an ionospheric outflow.

We modeled the effects of XUV flux on the ionosphere by coupling the ion hydrodynamics of the Polar Wind Outflow Model (PWOM) to the latest version of the SuperThermal Electron Transport (STET) code (Glocer et al. 2009; Glocer et al. 2012; Khazanov 2011; Khazanov et al. 2015). Full details of the model coupling will appear in a separate publication (Glocer et al. 2016). The XUV fluxes from the evolving Sun are expressed in terms of the total XUV flux, $\mathrm{F}_{0}$, of the Sun at the average level of magnetic cycle. We find that the photoelectron flux increases approximately linearly with the input XUV flux. We then used PWOM to calculate the ionized atmospheric escape rates along an open single magnetic field line of the polar region at heights between $200 \mathrm{~km}$ and $6000 \mathrm{~km}$. 


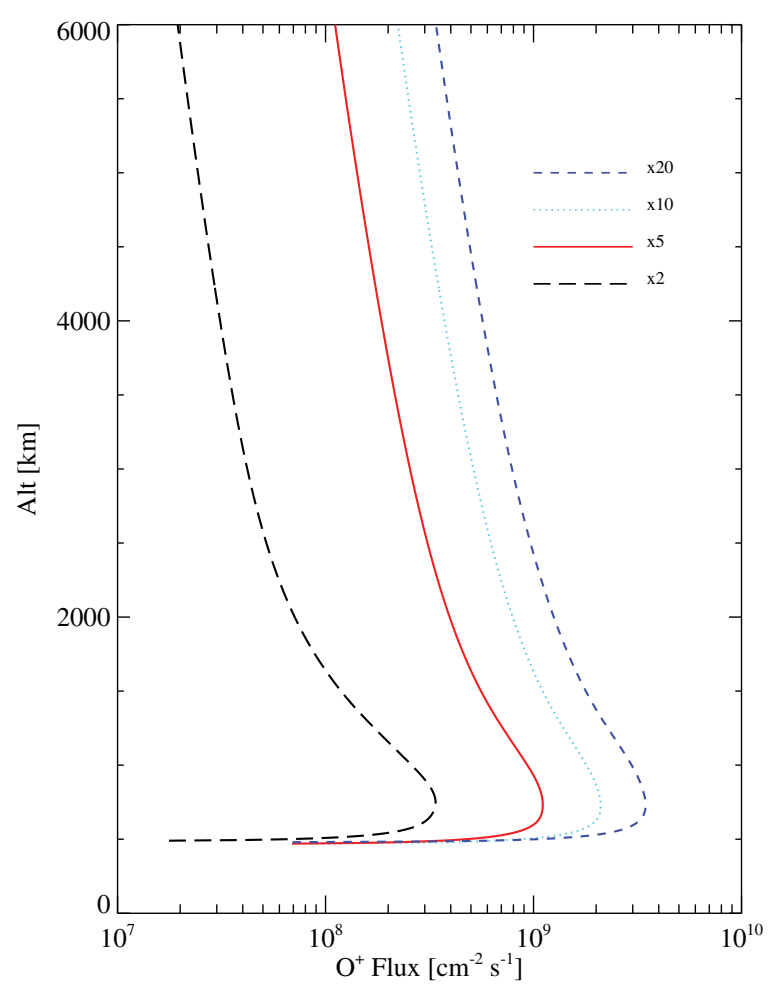

Figure 4. The mass loss rate of oxygen ions from the Earth atmosphere due to XUV irradiation from the young Sun at F=2 (long dash), 5 (dash-dot), 10 (dot), 20 (short dash).

The XUV flare flux at 10F0 corresponds to the associated super-Carrington type CME event. We then calculated the steady state outflow rate of $\mathrm{O}+$ ions driven by the input XUV flux and the value of the neutral temperature specified at the exospheric base at $200 \mathrm{~km}$. In order to evaluate the effect of the base temperature on the $\mathrm{O}^{+}$outflow rate, we calculated two escape models for the XUV flux of $10 \mathrm{~F}_{0}$ for these two exobase temperatures. We find that as we increase the base temperature by a factor of 2 (from $1000 \mathrm{~K}$ to $2000 \mathrm{~K}$ ), the resulting $\mathrm{O}+$ outflow rates increase by a factor of 10 . The total loss rate of $\mathrm{O}+$ at $\mathrm{h}=1000 \mathrm{~km}$ is found from the integration of this value over the whole area. Figure 4 shows that the mass loss of oxygen ions increases roughly linearly with the solar flux and reaches $400 \mathrm{~kg} / \mathrm{s}$ for $\mathrm{F}=10 \mathrm{~F}_{0}$ (Airapetian et al. 2017a). This estimate does not account for a number of effects typically contributing to the ion escape during space weather events associated with large solar flares. This mass loss rate can also be affected by precipitated energetic electrons from the day and night sides of the Earth magnetosphere. This input efficiently produces secondary superthermal electrons due to collisional ionization of species in the ambient ionosphere (Strangeway et al. 2005) and needs further study.

Our simulations of the atmospheric escape suggests that if we account for the escape for nitrogen ions along with oxygen ions, then the upper limit of escape rate at $10 \mathrm{~F}_{0}$, characteristic of the Sun's flux 3.8 billion years ago, is $\sim 400 \mathrm{~kg} / \mathrm{s}$. This suggests that Earth could have lost half of its 1-bar atmosphere in 300 million years after the secondary atmosphere was formed on the early Earth. Given that the Earth had an intensive volcanic and tectonic activity, this suggests that the XUV impact on the Earth's habitability was 


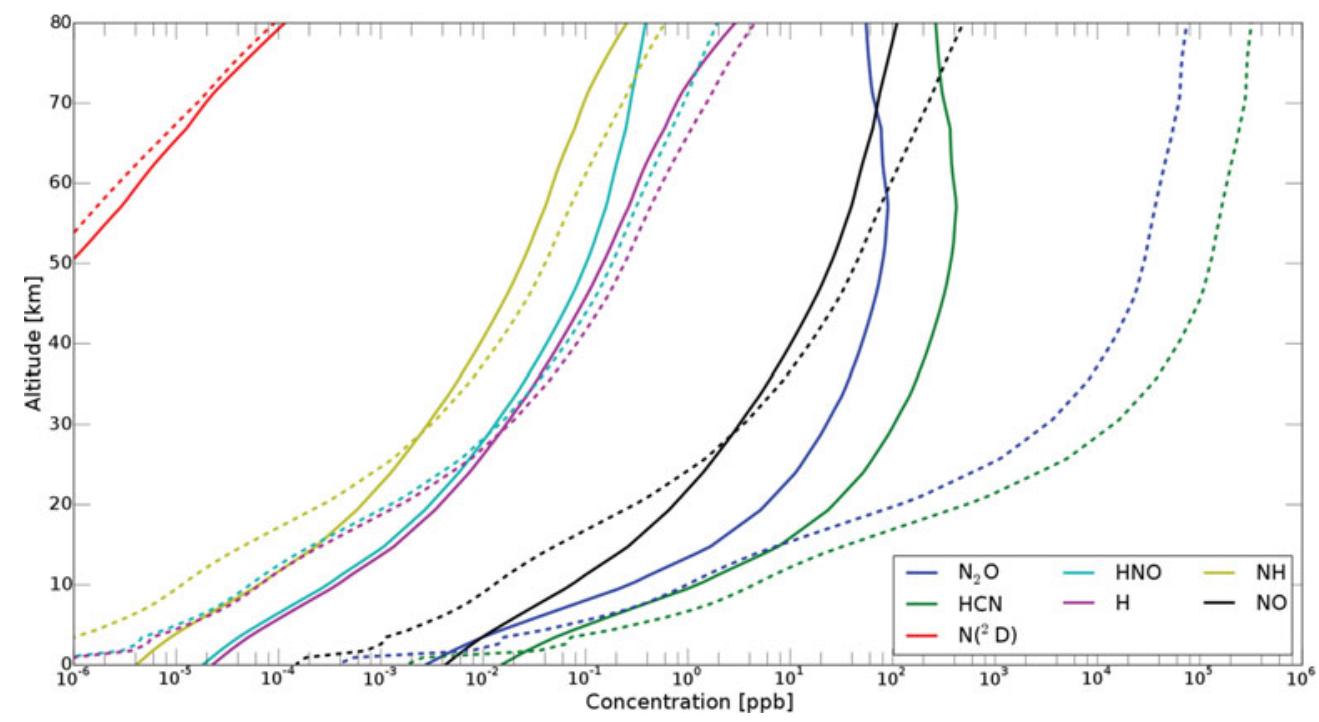

Figure 5. Aeroplanets model predictions of mixing ratios of species under chemical equilibrium driven by frequent energetic SEPs on the early Earth (Airapetian et al. 2016a).

pretty mild. This is consistent with the recent data suggesting that the atmosphere of the early Earth 2.7 Gyr ago was at least 0.5 bars (Som et al. 2016).

\section{Space Weather as a Factor of Life}

Our global magnetospheric simulations suggest that a disturbance of the early Earth magnetosphere by a super-Carrington CME event should shift the boundary of the openclosed field to $36^{\mathrm{deg}}$ in latitude, producing a polar cap opening $70 \%$ of the planet's dipole magnetic field. Thus, extended polar caps may provide a pathway for energetic electrons and protons accelerated in CME-driven shocks to penetrate the Earth atmosphere along the open field lines (Airapetian et al. 2016a).

The secondary atmosphere of the early Earth at 0.5 Gyr was nitrogen rich (80-90\%) and $\mathrm{CO}_{2}$ rich (10-20\%) with traces of methane, $\mathrm{CH}_{4}$, and water vapor, $\mathrm{H}_{2} \mathrm{O}$. Molecular nitrogen was mostly supplied by tectonic activity from the highly oxidized mantle wedges driven by subduction processes, while carbon dioxide, methane and water vapor were released by intensive volcanic activity (Mikhail \& Sverjensky 2014). We have recently applied our Aeroplanet model (Gronoff et al. 2014) to simulate the atmospheric chemistry of such highly reduced nitrogen-dominated $\left(79 \% \mathrm{~N}_{2}, 20 \% \mathrm{CO}_{2}, 0.4 \% \mathrm{CH}_{4}, 1 \%\right.$ $\mathrm{H}_{2} \mathrm{O}$ ) prebiotic Earth atmosphere at a surface pressure of 1 bar with the photochemistry controlled by the EUV-XUV flux fro the young Sun and a proton energy flux of 50 times that of the Jan 20, 2005 SEP event (Airapetian et al. 2016).

The Aeroplanets model calculates photoabsorption of the EUV-XUV flux from the early Sun and electron and proton fluxes to compute the corresponding energetic fluxes at all altitudes between $200 \mathrm{~km}$ to the surface (Gronoff et al. 2014). These fluxes are then used to calculate the photo and particle impact ionization/dissociation rates of the atmospheric species producing secondary electrons due to ionization processes. Then, using the XUV flux and the photoionization-excitation- dissociation cross-sections, we calculated the production of ionized and excited state species and resulted photoelectrons. In this steady-state model of the early Earth atmosphere, energetic protons from an SEP event precipitate into the middle and lower atmosphere (stratosphere, mesosphere and 
troposphere) and produce ionization, dissociation, dissociative ionization, and excitation of atmospheric species. The destruction of $\mathrm{N}_{2}$ into reactive nitrogen, $\mathrm{N}(2 \mathrm{D})$ and $\mathrm{N}(4 \mathrm{~S})$ and the subsequent destruction of $\mathrm{CO}_{2}$ and $\mathrm{CH} 4$ produces $\mathrm{NOx}, \mathrm{CO}$ and $\mathrm{NH}$ in the polar regions of the atmosphere as shown in Figure $5 . \mathrm{NO}_{x}$ then converts in the stratosphere to $\mathrm{NO}_{3}, \mathrm{HNO}_{2}$ and $\mathrm{HNO}_{3}$.

One of the major predictions of our atmospheric model is efficient production of nitrous oxide, $\mathrm{N}_{2} \mathrm{O}$, which is a potent greenhouse gas (Airapetian et al. 2016). This could represent a pathway to the resolution of the faint Young Sun's (FYS) paradox that suggest that the energy from faint young Sun would be insufficient to support liquid water on the early Earth contrary to geological evidence of its presence that time (Sagan and Mullen 1972; Ramirez 2016). The proposed models of the atmospheric warming due to large atmospheric concentration of $\mathrm{CO}_{2}, \mathrm{H}_{2} \mathrm{O}$ and/or $\mathrm{CH}_{4}$ cannot resolve the FYS paradox (Kasting 2010; Rosing et al. 2010). This problem becomes even worse for the Martian atmosphere that would require up to 4 bars of the atmospheric abundance of CO (Ramirez et al. 2014). Our model proposes a resolution of the FYS paradox due to collisional dissociation of the atmospheric $\mathrm{N}_{2}, \mathrm{CO}_{2}, \mathrm{CH}_{4}$ and $\mathrm{NH}_{3}$ producing abundant $\mathrm{NOx}$ and $\mathrm{NH}$ molecules and efficient formation of $\mathrm{N}_{2} \mathrm{O}$ through $\mathrm{NO}+\mathrm{NH} \rightarrow \mathrm{N}_{2} \mathrm{O}+$ $\mathrm{H}$ (Airapetian et al. 2016b). Atmospheric $\mathrm{N}_{2} \mathrm{O}$ density reaches a concentration with the mixing ratio of 0.3 to 1 ppmv in the lower atmosphere depending of availability of gases shielding nitrous oxide from photodestruction. The sources and sinks for $\mathrm{N}_{2} \mathrm{O}$ depend strongly on the chemical composition of the initial atmosphere and the energy flux in accelerated protons. Specifically, our simulations show that $\mathrm{N}_{2} \mathrm{O}$ 's abundance increases with increasing $\mathrm{CO}_{2} / \mathrm{CH}_{4}$ ratio in the initial atmosphere. Moreover, the derived value should be considered as a lower bound, because our model does not account for a number of factors including eddy diffusion and convection effects, concentration of hazes, inclusion of $\mathrm{SO}_{2}$ and $\mathrm{H}_{2} \mathrm{~S}$ volcanic outgassing sources and Rayleigh scattering of solar EUV radiation that significantly reduces photodestruction of $\mathrm{N}_{2} \mathrm{O}$, and therefore increases its production. Indeed, Earth's atmospheric data suggest that stratospheric-tropospheric exchange provide flat vertical profiles of ${ }^{7} \mathrm{Be}$ and ${ }^{1} 0 \mathrm{Be}$ from $30 \mathrm{~km}$ to $2-3 \mathrm{~km}$ above the ground at higher lattitudes (60-90 deg) (Land \& Feichter 2003). Thus, we expect the same profiles for $\mathrm{N}^{2} \mathrm{O}$ and $\mathrm{HCN}$ vertical profiles. This will provide the mixing ratio of $\mathrm{N}_{2} \mathrm{O}$ at least $1 \mathrm{ppm}$ in the lower troposphere required to provide efficient greenhouse warming. Also, energetic protons associated with SEP events significantly enhance atmospheric ion production rates, which in turn that drive increased rate of formation/nucleation of newly formed and/or existing production of stratospheric aerosol particles by up to one order of magnitude in the polar regions at $10-25 \mathrm{~km}$, which provides an efficient shield from UV emission around $240 \mathrm{~nm}$ (Mironova \& Usoskin 2014).

Greater typical energies of SEP events from the young Sun could be another factor that contribute to the increased production rate by a factor of 5-10 greater than that conservatively assumed in our model. This is due to the fact that young Sun's corona that represents the source of CMEs was at least by a factor of 10 denser as compared to the current Sun. Thus, denser corona provided correspondingly larger concentration of seed particles that participated in acceleration processes driven by CME driven shock waves closer to the solar surface. Also, our recent simulations suggest that the particle acceleration via diffusive shock acceleration mechanisms on a quasi-parallel shocks produce mostly SEPs with harder spectrum (Airapetian et al. 2017b) similar to the Feb 1956 SEP event (see Figure 6). Also, recent statistical study of These spectra suggests the particle flux at $1 \mathrm{GeV}$ by 2 orders of magnitude greater than that assumed in the current model by Airapetian et al. 2016. Because the production of reactants is proportional to the number of incoming particles, it should be linearly scaled for the incident 


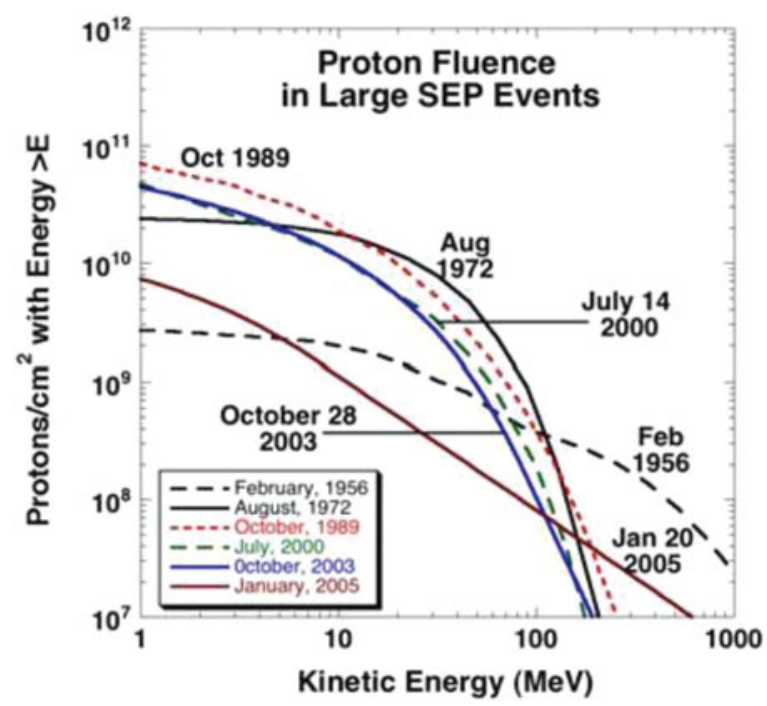

Figure 6. Protons fluence spectra from the largest SEPs observed over the last 50 years (Mewaldt et al. 2005).

flux. Thus, the concentration of produced $\mathrm{N}_{2} \mathrm{O}$ and $\mathrm{HCN}$ should be boosted by a factor of 100 at $10-15 \mathrm{~km}$.

Recent statistics of CMEs associated with SEP events shows that SEPs with hardest particle fluence spectra are correlated with CMEs with large initial acceleration when they were observed close to the Sun (Gopalswamy et al. 2016). Large initial acceleration of CMEs suggests that they were originated from structures with stronger magnetic field strength that rapidly inflate producing strong shock waves in the outer solar corona. These shocks then provide a fertile ground for particle acceleration in SEP events. This empirical picture is consistent with our recent simulations of SEP accelerated events from the young Sun formed from the strong shocks produced by magnetized CMEs via diffusive shock acceleration mechanisms on a quasi-parallel shocks produce mostly SEPs with harder fluence spectra (Airapetian et al. 2017a) similar to the Feb 1956 SEP event.

Such hard particle spectra suggest the particle flux at $1 \mathrm{GeV}$ which is by a factor of 20 at $600 \mathrm{MeV}$ greater than the Jan 202005 SEP (see Figure 6) event taken in our recent prebiotic chemistry model of the early Earth (Airapetian et al. 2016a). Because the production rates of $\mathrm{N}_{2} \mathrm{O}$ and $\mathrm{HCN}$ is proportional scaled with the number of incoming protons at $1 \mathrm{GeV}$, we should therefore scale the produced concentration of these molecules in the lower stratosphere (at $20 \mathrm{~km}$ ) by a factor of 30 larger than discussed in our paper. Thus, the concentration of produced N2O and HCN should be boosted by a factor of 100 in the Earth's lower atmosphere. Thus, the combination of discussed factors would boost the concentration of $\mathrm{N}_{2} \mathrm{O}$ to the level of 100 ppmv. Climate models by Roberson et al. 2011 of the for the Proterozoic Earth with the concentration of methane at $1.6 \mathrm{ppm}$ at the fixed concentration of carbon dioxide at $320 \mathrm{ppm}$ yield surface warming above $0^{0} \mathrm{C}$. This suggest that our model presents an opportunity to obtain temperatures above the freezing point in our ongoing 3D GCM simulations, and thus resolve the longstanding Faint Young Sun paradox for the early Earth and Mars. 


\section{Conclusions}

As we discussed in this paper, space weather effects from the young Sun can contribute to the habitability conditions on the early Earth in a variety of ways. First, we have shown that the high magnitude southward IMF and large dynamic pressures from super Carrington type CME events can restructure the Earth's magnetosphere due to reconnection events and widen its polar caps. Then, the dissipation of large induced geomagnetic currents can heat the thermospheric plasma to high temperature that can support its escape from the planet in its earliest phase of evolution when CME events were frequent and energetic. Second, high XUV fluxes from associated superflare flare events can support the escape of oxygen and possibly nitrogen ions due to production of photoelectrons. However, in our models, where the escape process was uncoupled with the thermal effects of inflated exosphere due, and therefore, they provide the lower bound of escape rates. In these ways, CMEs can provide negative conditions for habitability on the early Earth and Mars especially in the first 0.5 billion years of the Sun's magnetically active phase of evolution. The conditions on the early Mars were more severe because of much lower surface gravity and the efficiency of photochemical escape via dissociative recombination of $\mathrm{O} 2$ and possibly $\mathrm{N} 2$ producing hot atomic oxygen and nitrogen.

However, frequent SEP events from the young Sun probably played a positive role in setting the conditions for formation of hydrogen cyanide and nitrous oxide in the lower stratosphere and upper troposphere of the early Earth and Mars. Organic molecules may subsequently rain out into surface reservoirs and ignited higher order chemistry producing more complex organics. For example, the hydrolysis of HCN produces formamide, $\mathrm{HCONH}_{2}$. When irradiated with energetic protons, formamide can serve as a precursor of complex biomolecules that are capable of producing amino acids, the building blocks of proteins and nucleobases, sugars and nucleotides, the constituents of RNA and DNA molecules (Saladino et al. 2015). In our recent experiments, irradiation of gas mixture resembling the young Earth atmosphere with high-energy protons ( $2.5 \mathrm{MeV}$ ) produced amino acids including glycine and alanine (Kobayashi et al. 2001; 2017). The irradiation of the same mixture by the spark discharge (accelerated electrons) or UV irradiation (2500/AA), produced no amino acids at $\mathrm{CH}_{4}$ mixing ratio (less than $15 \%$ ). Thus, considering fluxes of various energies on the primitive Earth, energetic protons appear to be an efficient factor to produce N-containing organics than any other conventional energy sources like thundering or solar UV emission irradiated the early Earth atmosphere. Also, abiotic production of nitrous oxide in the lower troposphere at $10-20 \mathrm{ppm}$ driven by energetic protons can provide an efficient way to resolve a long standing Faint Young Sun paradox to explain the warming of our young planet to keep water in the liquid state in its early history (Airapetian et al. 2016; Airapetian et al. 2017b).

In our future work, we plan to develop a comprehensive $1 \mathrm{D}$ and $2 \mathrm{D}$ photo-collisional models of the early Earth that will describe the production of greenhouse gases and biological molecules in the troposphere with implementation newly develop models of solar energetic particle events from the young Sun as inputs along with more realistic representation of volcanic gasses and aerosols in the early Earth atmosphere. These models will provide insights in understanding the challenging problem of warming of early Mars and also expand the definition of habitability zones around main-sequence $\mathrm{G}$ and $\mathrm{K}$ stars with volcanically active planets.

\section{References}

Abramov, O. \& Mojzsis, S. J. 2009, Nature, 459, 419 
Airapetian, V. S., Glocer, A., Khazanov, G. V., Loyd, R. O. P., France, Kevin et al. 2017a, ApJ Letters, 836, L3

Airapetian, V. S., Zank, G., Verkhodlyadova, O. Li, G., Gronoff, G. 2017b, in preparation.

Airapetian, V. S., Glocer, A., Gronoff, G., E. H/ebrard, E., Danchi, W. 2016, Nature Geoscience, DOI:10.1038/NGEO2719

Airapetian, V. \& Usmanov, A. 2016, ApJ Let., 817, L24

Airapetian, V., Glocer, A., \& Danchi, W. 2015, Proc. of the 18th Cambridge Workshop on Cool Stars, Stellar Systems, and the Sun, Lowell Observatory (9-13 June 2014) Eds: G. van Belle \& H. Harris (eprint arXiv:1409.3833)

Aarnio, A. N., Stassun, K. G., Hughes, W. J., \& McGregor, S. L. 2011, Sol.Phys., 268, 195

Thakur, N., Gopalswamy, N., Xie, H., Makela, P., Akijama, P., Davila, J. M., \& Rickard, L. J. 2014, ApJLet, 790, L13

Birch, J. L. et al. 2016, Science, 352, id.aaf2939

Biggin, A. J., Strik, G. H. M. A., \& Langereis, C. G., 2008, Nature Geoscience 1, 395

Bell, E. A., Boehnke, P., Harrison, T.M. \& Mao, W. L. 2015, PNAS, 112, 14,518

Claire, M. W. et al. 2012, ApJ. 757, 95

Cohen, O., Drake, J. J., Glocer, A., Garraffo, C., Poppenhaeger, K., Bell, J. M., Ridley, A. J., Gombosi, T. I. 2014, ApJ, 790, 57

Garraffo, C., Drake, J. J., Cohen, O. 2016, A\& A, 595, id.A110

Gomes, R., Levison, H. F., Tsiganis, K., \& Morbidelli, A. 2005, Nature, 435, 466

de Zeeuw, D. L., Sazykin, S., Wolf, R. A., Gombosi, T. I., Ridley, A. J., Toth, G. 2004, JGR, 109, A12

Emslie, A. G. et al. 2012, ApJ, 759, 71

Glocer, A., G. Toth, T. Gombosi, \& D. Welling, 2009, JGR., 114

Glocer, A., N. Kitamura, G. Tóth, \& T. Gombosi, 2012, JGR., 117, A04318, doi: 10.1029/2011JA017136

Gopalswamy, N. 2011, First Asia-Pacific Solar Physics Meeting ASI Conference Series, 2011, Vol. 2, pp 2, Edited by Arnab Rai Choudhuri \& Dipankar Banerjee

Gopalswamy, N., Yashiro, S., Thakur, N., Mäkelä, P., Xie, H., \& Akiyama, S. 2016, ApJ, 833, Issue 2 , article id. 216

Gough, D. O. 1981, Solar Phys. 74, 21

Gronoff, G. et al. 2014, Geophys. Res. Lett. 41, 4844

Güdel, M, Guinan, E. F., \& Skinner, S. L. 1997, ApJ, 483, 947

Kasting, J. F. 2010, Nature 464, 687

Knipp, D. J., Pette, D. V., Lilcommons, L. M. et al. 2017, Space Weather, 15, doi:10.1002/20165W001567

Khazanov, G. 2011, Kinetic Theory of the Inner Magnetospheric Plasma, Astrophysics and Space Science Library, Vol. 372. ISBN 978-1-4419-6796-1. Springer

Khazanov, G. V., Tripathi, A. K., Sibeck, D., Himwich, E., Glocer, A., \& Singhal, R. P. 2015, $J G R, 20$, Issue 11, 9891

Kobayashi, K., Masuda, H., Ushi, K., Ohashi, A. Yamanashi, H. et al. 2001, Adv. Space Res. 27, No. 2, 207

Kobayashi, K,. Aoki, R., Abe, H., Kebukawa, Y., Shibata, H., Yoshida, S., Fukuda, H., Kondo, K., Oguri, Y., \& Airapetian, V. S. 2017, Astrobiology Science Conference 2017, Abstract \#3 259

Kramar, M., Airapetian, V., \& Lin, H. 2016, Frontiers in Astronomy and Space Sciences, Volume 3 , id. 25

Lammer, H., Kasting, J. F., Chassefiere, Johnson, R. E., Kulikov, Y. N., \& Fian, F. 2008, Space Sci. Rev., 139, 399

Land, C. \& Feichter, J. 2003, JGR, 108, Issue D12, 10.1029/2002JD002543

Le et al. 2016, GRL, 43, 2396

Love, J. J. 2012, GRL., 39, L10301

Loyd, R. O. P., France, Kevin, Youngblood, A., Schneider, C. Brown, A., Hu, R., Linsky, K., Froning, C. S., Redfield, S., Rugheimer, S., \& Tian, F. 2016, ApJ, 824, 102

Maehara, H. et al. 2012, Nature 485, 478

Miyake, F., Nagaya, K., Masuda, K., \& Nakamura, T. 2012, Nature, 486, 240 
Ngwira, C. M., Pulkkinen, A., Kuznetsova, M. M. \& Glocer, A. 2014, JGR, 119, 4456

Nitta, N. V., Aschwanden, M. J., Freeland, S. L., Lemen, J. R., Wülser, J.-P., \& Zarro, D. M. 2014, Sol. Phys., 289, 1257

Mikhail \& Sverjensky 2014, Nature Geoscience 7, 816

Mewaldt, R. A., Cohen, C. M. S., Labrador, A. W., Leske, R. A., Mason, G. M., Desai, M. I., Looper, M. D., Mazur, J. E., Selesnick, R. S., \& Haggerty, D. K. 2005, JGR, 110, A09S18

Mironova, I. A. \& Usoskin, I. G. 2014, Environ. Res. Lett., 9

Powell, K. G., Roe, P. L., Linde, T. J., Gombosi, T. I., \& De Zeeuw, D. L. 1999, JCP, 154, 284

Pevtsov, A. A., Fisher, G. H., Acton, L. W., Longcope, D. W., Johns-Krull, C. M., Kankelborg, C. C., \& Metcalf, T. R. 2003, ApJ, 589, 1387

Ramirez, R. M., Kopparapu, R., Zugger, M. E., Robinson, T. D., Freedman, \& R. Kasting, J. 2014, Nature Geoscience, 7, Issue 1, 59

Ramirez, R. M. 2016, Nature Geoscience, 9, Issue 6, 413

Ridley, A. J., Hansen, K. C., Tóth, G., de Zeeuw, D. L., Gombosi, T. I., \& Powell, K. G. 2002, JGR, 107, 1290

Riley, P., Caplan, R. M., Giacalone, J., Lario, D., \& Liu, Y. 2016, ApJ, 819, 57

Roberson, A. L., Roadt, J., Halevy, I., \& Kasting, J. F. 2011, Geobiology, 9, 313

Rosing, M. T., Bird, D. K., Sleep, N. H. \& Bjerrum, C. J. 2010, Nature 464, 744

Sagan, C. \& Mullen, G. 1972, Science, 177, 52

Saladino, R., Carota, E., Botta, G., Kapralov, M., Timoshenko, G. N., Rozanov, A. Y., Krasavin, E., \& Ernesto Di Mauro, E. 2015, Publ. Nat. Acad. Sci., 112 (21), E2746

Schrijver, C. J., Kauristie, Kirst A., Alan D., Denardini, C. M., Gibson, S. E., Glover, A., Gopalswamy, N., Grande, M., Hapgood, M., Heynderickx, D., \& 16 coauthors, 2015, Advances in Space Res., 55, 2745

Shibayama, T., Maehara, H., Notsu, S., Notsu, Y., Nagao, T., Honda, S., Ishii, T. T., Nogami, D., \& Shibata, K. 2013, ApJSS, 209, 5

Schrijver, C. J. \& Title, A. M. 2013, ApJ, 619, 1077

Som, S. M., Buick, R., Hagadorn, J. W., Blake, T. S., Perreault, J. M., Harnmeijer, J. P., \& Catling D. C. 2016 Nature Geoscience, 9,448

Sterenborg, M. G., Cohen, O. , Drake, J. J., \& Gombosi, T. I. 2011, 116, A01217

Strangeway, R. J., Ergun, R. E., Su, Y.-J., Carlson, C. W., \& Elphic, R. C. 2005, JGR, 110, Issue A3,CiteID A03221

Tarduno, J., A., Blackman, E. G., \& Mamajek, E. E. 2014, Physics of the Earth and Planetary Interiors, 233, 68

Tarduno, J. A., Cottrell, R. D., Watkeys, M. K., Hofmann, A., Doubrovine, P. V., Mamajek, E. E., Liu, D., Sibeck, D. G., Neukirch, L. P., \& Usui, Y. 2010, Science, 327, Issue 5970, 1238

Tian, F., Kasting, J. F. Liu, H.-Li, \& Roble, R. G. 2008, JGR, 113, Issue E5, CiteID E05008

Thomas, B. C., Melott, A. L., Arkenberg, K. R., \& Snyder, B. R. 2013, Geoph. Res. Let, 40, 1237

Tsurutani, B. T., Gonzales, W. D., Lakhina, G. S. \& Alex, S. 2003, J. Geophys. Res. 108, 1268

Tsurutani, B. T. \& Lakhina, G. S. 2014, Geoph. Res. Let, 41, 287

Tu, L., Johnstone, C., Gudel, M., \& Lammer, H.2016, A\& A, 577, L3

Vidotto, A. A., Gregory, S. G., Jardine, M., Donati, J. F., Petit, P. et al. 2014, NMRAS, 441, 2361

Yashiro, S. \& Gopalswamy, N. 2009, in IAU Symp.257, Universal Geophysical Processes, ed. N. Gopalswamy \& D. F. Webb (Cambridge: Cambridge Univ. Press), 233

Weimer, D. R., Mlynczak, M. G., Hunt, L. A., \& Tobiska, W. K. 2015, JGR, 120, 5998

Wilde, S., Valley, J. W., Peck, W. H. \& Graham, C. M. 2001, Nature, 409, 175

Wood, B. E., Müller, H-R, Zank, G. P., Linsky, J. L.\& Redfield, S. 2005, ApJ, 628, L143

Zhao, Y., Wang, R., Lu, Q., Du, A., Yao, Z., \& Wu, M. 2016, JGR, 121, Issue 11, 10,898 